\title{
HILBERT 2-CLASS FIELD TOWERS OF INERT IMAGINARY QUADRATIC FUNCTION FIELDS
}

\author{
HWANYUP JUNG
}

\begin{abstract}
In this paper we study the infiniteness of Hilbert 2-class field towers of inert imaginary quadratic function fields over $\mathbb{F}_{q}(T)$, where $q$ is a power of an odd prime number.
\end{abstract}

\section{Introduction And Statement of the Results}

Let $k=\mathbb{F}_{q}(T)$ be a rational function field over the finite field $\mathbb{F}_{q}$ of $q$ elements, $\infty=(1 / T)$ and $\mathbb{A}=\mathbb{F}_{q}[T]$. For any finite separable extension $F$ of $k$, write $\mathcal{O}_{F}$ for the integral closure of $\mathbb{A}$ in $F$ and $H_{F}$ for the Hilbert class field of $F$ with respect to $\mathcal{O}_{F}$ (see [4]). Let $\ell$ be a prime number. Let $F_{0}^{(\ell)}=F$ and $F_{n+1}^{(\ell)}$ be the Hilbert $\ell$-class field of $F_{n}^{(\ell)}$ for $n \geq 0$, i.e., $F_{n+1}^{(\ell)}$ is the maximal extension of $F_{n}^{(\ell)}$ inside $H_{F_{n}^{(\ell)}}$ whose degree over $F_{n}^{(\ell)}$ is a power of $\ell$. The sequence of fields

$$
F=F_{0}^{(\ell)} \subset F_{1}^{(\ell)} \subset \cdots \subset F_{n}^{(\ell)} \subset \cdots
$$

is called the Hilbert $\ell$-class field tower of $F$. We say that the Hilbert $\ell$-class field tower of $F$ is infinite if $F_{n}^{(\ell)} \neq F_{n+1}^{(\ell)}$ for each $n \geq 0$. Let $\mathcal{C} l_{F}$ and $\mathcal{O}_{F}^{*}$ be the ideal class group and the group of units of $\mathcal{O}_{F}$, respectively. For any multiplicative abelian group $A$, write $r_{\ell}(A)=\operatorname{dim}_{\mathbb{F}_{\ell}}\left(A / A^{\ell}\right)$ for the $\ell$-rank of $A$. Schoof [6] has shown that if $r_{\ell}\left(\mathcal{C} l_{F}\right) \geq 2+2 \sqrt{r_{\ell}\left(\mathcal{O}_{F}^{*}\right)+1}$, then the Hilbert $\ell$-class field tower of $F$ is infinite. This is a function field analog of Golod-Shafarevich.

Now, we assume that $q$ is odd with $q \equiv 3 \bmod 4$. In $[2,3]$, we study the infiniteness of Hilbert 2-class field towers of ramified imaginary or real quadratic function fields over $k$. The aim of this paper is to study the infiniteness of Hilbert 2-class field towers of inert imaginary quadratic function fields over $k$. Let $F$ be an inert imaginary

Received by the editors October 30, 2012. Accepted April 4, 2013.

2010 Mathematics Subject Classification. 11R11, 11R58.

Key words and phrases. Hilbert 2-class field tower, inert imaginary, quadratic function field.

The author is supported by the National Research Foundation of Korea (NRF) funded by the Ministry of Education, Science and Technology (NRF-2010-0008139). 
quadratic function field over $k$, i.e., $F$ is a quadratic extension of $k$ in which $\infty$ is inert. Fix a generator $\gamma$ of $\mathbb{F}_{q}^{*}$. Let $\mathcal{P}$ be the set of monic irreducible polynomials in $\mathbb{A}$. Then $F$ can be written as $F=k(\sqrt{D})$ with $D=\gamma P_{1} \cdots P_{t}, P_{i} \in \mathcal{P}$ for $1 \leq i \leq t$ and $2 \mid \operatorname{deg} D$. Here, $D$ is uniquely determined by $F$ and write $D_{F}=D$. We say that $D_{F}$ is special if $2 \mid \operatorname{deg} P_{i}$ for all $1 \leq i \leq t$. For $0 \neq N \in \mathbb{A}$, write $\omega(N)$ for the number of distinct monic irreducible divisors of $N$. It is known ([1, Corollary $3.5])$ that $r_{2}\left(\mathcal{C} l_{F}\right)$ is equal to $\omega\left(D_{F}\right)$ or $\omega\left(D_{F}\right)-1$ according as $D_{F}$ is special or non-special. Since $\mathcal{O}_{F}^{*}=\mathbb{F}_{q}^{*}$ (i.e., $r_{2}\left(\mathcal{O}_{F}^{*}\right)=1$ ), by Schoof's Theorem, $F$ has infinite Hilbert 2-class field tower if $r_{2}\left(\mathrm{Cl}_{F}\right) \geq 5$.

For an inert imaginary quadratic function field $F$ over $k$, let $s_{F}$ be the number of monic irreducible divisors of $D_{F}$ of odd degree. Since $\operatorname{deg} D_{F}$ is even, $s_{F}$ is a nonnegative even integer. Let $\vartheta_{F}$ be 1 or 0 according as $s_{F}=0$ or $s_{F} \geq 2$. Let $r_{4}\left(\mathcal{C} l_{F}\right)=r_{2}\left(\mathcal{C} l_{F}^{2}\right)$ be the 4-rank of $\mathcal{C} l_{F}$. Then we have

Theorem 1.1. Assume that $q \equiv 3 \bmod 4$. Let $F$ be an inert imaginary quadratic function field over $k$. If $r_{4}\left(\mathcal{C} l_{F}\right) \geq 3+\vartheta_{F}$, then the Hilbert 2-class field tower of $F$ is infinite.

For any positive even integer $n$ and integers $r, s$ with $0 \leq s \leq r$, let $Z_{r ; n}$ be the set of inert imaginary quadratic function fields $F$ with $r_{2}\left(\mathcal{C l}_{F}\right)=r$ and $\operatorname{deg}\left(D_{F}\right)=n$, and $Z_{r, s ; n}$ be the subset of $Z_{r ; n}$ consisting of $F \in Z_{r ; n}$ with $r_{4}\left(\mathcal{C} l_{F}\right)=s$. Let $Z_{r, s ; n}^{*}$ be the subset of $Z_{r, s ; n}$ consisting of $F \in Z_{r, s ; n}$ whose Hilbert 2-class field tower is infinite. We define a density $\varrho_{r, s}^{*}$ by

$$
\varrho_{r, s}^{*}=\liminf _{\substack{n \rightarrow \infty \\ n: \text { even }}} \frac{\left|Z_{r, s ; n}^{*}\right|}{\left|Z_{r ; n}\right|} .
$$

Then we have

Theorem 1.2. Assume that $q \equiv 3 \bmod 4$. We have $\varrho_{3, s}^{*} \geq 2^{-3}\left(2^{3}-1\right)^{-1}$ for $s=1,2$ and $\varrho_{4, s}^{*} \geq 2^{-6}\left(2^{4}-1\right)^{-1}$ for $0 \leq s \leq 3$.

We remark that Theorem 1.2 means that a positive proportion of inert imaginary quadratic function fields $F$ with $r_{2}\left(\mathcal{C} l_{F}\right)=r$ have infinite Hilbert 2-class field towers and $r_{4}\left(\mathcal{C l} l_{F}\right)=s$ for $r=3, s=1,2$ or $r=4,0 \leq s \leq 3$.

\section{Preliminaries}

2.1. Rédei-matrix and 4-rank of class group Let $F$ be an inert imaginary quadratic function field over $k$ with $D_{F}=\gamma P_{1} \cdots P_{t}$. Let $d_{i} \in \mathbb{F}_{2}$ be defined by 
$d_{i} \equiv \operatorname{deg} P_{i} \bmod 2$ for $1 \leq i \leq t$. Let $M_{F}=\left(e_{i j}\right)_{1 \leq i, j \leq t}$ be a $t \times t$ matrix over $\mathbb{F}_{2}$, where $e_{i j} \in \mathbb{F}_{2}$ is defined by $\left(\frac{P_{i}}{P_{j}}\right)=(-1)^{e_{i j}}$ for $1 \leq i \neq j \leq t$ and the diagonal entries $e_{i i} \in \mathbb{F}_{2}$ are defined to satisfy the relation $d_{i}=\sum_{j=1}^{t} e_{i j}$. We associate a $(t+1) \times t$ matrix $R_{F}$ over $\mathbb{F}_{2}$ to $F$ as follows:

- If $D_{F}$ is non-special, $R_{F}$ is the $(t+1) \times t$ matrix obtained from $M_{F}$ by adding $\left(d_{1} \cdots d_{t}\right)$ in the last row.

- If $D_{F}$ is special, $R_{F}$ is the $(t+1) \times t$ matrix obtained from $M_{F}$ by adding $\left(\begin{array}{lll}e_{B 1} & \ldots & e_{B t}\end{array}\right)$ in the last row, where $B$ is a monic polynomial in $\mathbb{A}$ such that $(B)=N_{F / k}(\mathfrak{B}), \mathfrak{B}^{\sigma-1}=(x)$ with $N_{F / k}(x) \in \mathbb{F}_{q}^{*} \backslash \mathbb{F}_{q}^{* 2}$ and $e_{B i} \in \mathbb{F}_{2}$ is defined by $\left(\frac{B}{P_{i}}\right)=(-1)^{e_{B i}}$. (Here, $\sigma$ is the generator of $\operatorname{Gal}(F / k)$.)

Then we have ([1, Corollary 3.8])

$$
r_{4}\left(\mathcal{C} l_{F}\right)=\omega\left(D_{F}\right)-\operatorname{rank} R_{F} .
$$

2.2. Some lemmas Let $E$ and $K$ be finite geometric separable extensions of $k$ such that $E / K$ is a cyclic extension of degree $\ell$, where $\ell$ is a prime number not dividing $q$. Let $S_{\infty}(K)$ be the set of primes of $K$ lying above $\infty$. Let $\gamma_{E / K}$ be the number of prime ideals of $\mathcal{O}_{K}$ that ramify in $E$ and $\rho_{E / K}$ be the number of primes $\mathfrak{p}_{\infty}$ in $S_{\infty}(K)$ that ramify or inert in $E$. In [2, Proposition 2.1], we have shown that the Hilbert $\ell$-class field tower of $E$ is infinite if

$$
\gamma_{E / K} \geq\left|S_{\infty}(K)\right|-\rho_{E / K}+3+2 \sqrt{\ell\left|S_{\infty}(K)\right|+(1-\ell) \rho_{E / K}+1} .
$$

Now, by using this result, we give some sufficient conditions for an inert imaginary quadratic function field $F$ to have infinite Hilbert 2-class field tower.

Lemma 2.1. Let $F$ be an inert imaginary quadratic function field over $k$. If there exists a nonconstant divisor $D^{\prime}$ of $D_{F}$ such that either $D^{\prime}$ or $D_{F} / D^{\prime}$ is monic of even degree and $\left(\frac{D^{\prime}}{P_{i}}\right)=1$ for monic irreducible divisors $P_{i}(1 \leq i \leq 4)$ of $D_{F}$, then $F$ has infinite Hilbert 2-class field tower.

Proof. Let $K=k\left(\sqrt{D^{\prime}}\right)$ and $E=K F$. Since $P_{1}, P_{2}, P_{3}$ and $P_{4}$ split in $K$, we have $\gamma_{E / K} \geq 8$. We also have $\left|S_{\infty}(K)\right|=\rho_{E / K}=2$ or $\left(\left|S_{\infty}(K)\right|, \rho_{E / K}\right)=(1,0)$ according as $D^{\prime}$ is monic of even degree or $D_{F} / D^{\prime}$ is monic of even degree. Then $E$ has infinite Hilbert 2-class field tower. It can be easily shown that $E$ is contained in $F_{1}^{(2)}$. Thus $F$ also has infinite Hilbert 2-class field tower.

Lemma 2.2. Let $F$ be an inert imaginary quadratic function field over $k$. If $D_{F}$ has two distinct nonconstant monic divisors $D_{1}$ and $D_{2}$ of even degrees satisfying 
$\left(\frac{D_{1}}{P_{i}}\right)=\left(\frac{D_{2}}{P_{i}}\right)=1$ for monic irreducible divisors $P_{i}(i=1,2)$ of $D_{F}$, then $F$ has infinite Hilbert 2-class field tower.

Proof. Let $K=k\left(\sqrt{D_{1}}, \sqrt{D_{2}}\right)$ and $E=K F$. Since $P_{1}, P_{2}$ and $\infty$ splits completely in $K, E$ is contained in $F_{1}^{(2)}$. Since $\gamma_{E / K} \geq 8$ and $\left|S_{\infty}(K)\right|=\rho_{E / K}=4$, we see that $E$ has infinite Hilbert 2-class field tower. Hence, $F$ also has infinite Hilbert 2-class field tower.

2.3. Some asymptotic results Let $\mathcal{P}$ be the set of all monic irreducible polynomials in $\mathbb{A}=\mathbb{F}_{q}[T]$. For positive integers $n$ and $t$, write $\mathcal{P}(n, t)$ for the set of monic square free polynomials $N \in \mathbb{A}$ with $\operatorname{deg} N=n$ and $\omega(N)=t$, and $\mathcal{P}^{\prime}(n, t)$ for the subset of $\mathcal{P}(n, t)$ consisting of $N=P_{1} \cdots P_{t} \in \mathcal{P}(n, t)$ such that $\operatorname{deg} P_{i} \neq \operatorname{deg} P_{j}$ for $1 \leq i \neq j \leq t$. Let $\mathcal{P}_{2}(n, t)$ be the subset of $\mathcal{P}(n, t)$ consisting of $N=P_{1} \cdots P_{t} \in \mathcal{P}(n, t)$ such that $\operatorname{deg} P_{i}$ is even for all $1 \leq i \leq t$ and $\mathcal{P}_{2}^{\prime}(n, t)=\mathcal{P}_{2}(n, t) \cap \mathcal{P}^{\prime}(n, t)$. As $n \rightarrow \infty$, we have

$$
\begin{aligned}
& |\mathcal{P}(n, t)|=\frac{q^{n}(\log n)^{t-1}}{(t-1) ! n}+O\left(\frac{q^{n}(\log n)^{t-2}}{n}\right), \\
& \left|\mathcal{P}_{2}(n, t)\right|=\frac{q^{n}(\log n)^{t-1}}{(t-1) ! 2^{t-1} n}+O\left(\frac{q^{n}(\log n)^{t-2}}{n}\right), \\
& \left|\mathcal{P}(n, t) \backslash \mathcal{P}^{\prime}(n, t)\right|=o\left(\frac{q^{n}(\log n)^{t-1}}{n}\right) .
\end{aligned}
$$

For $N=P_{1} \cdots P_{t}, M=Q_{1} \cdots Q_{t} \in \mathcal{P}^{\prime}(n, t)$, we say that $N$ and $M$ are equivalent if $\operatorname{deg} P_{i} \equiv \operatorname{deg} Q_{i} \bmod 2$ for $1 \leq i \leq t$ and $\left(\frac{P_{i}}{P_{j}}\right)=\left(\frac{Q_{i}}{Q_{j}}\right)$ for $1 \leq i<j \leq t$. Write $\mathcal{N}(N)$ for the set of polynomials in $\mathcal{P}^{\prime}(n, t)$ which are equivalent to $N$. Then we have ([2, Proposition 2.9])

$$
|\mathcal{N}(N)|=2^{1-\frac{\left(t^{2}+t\right)}{2}} \cdot \frac{q^{n}(\log n)^{t-1}}{(t-1) ! n}+O\left(\frac{q^{n}(\log n)^{t-2}}{n}\right)
$$

as $n \rightarrow \infty$.

For a positive even integer $n$ and a positive integer $t$, let $\mathcal{R}^{\prime}(n, t)=\mathcal{P}^{\prime}(n, t) \backslash$ $\mathcal{P}_{2}^{\prime}(n, t)$. Then we have ([3, Proposition 2.4])

$$
\left|\mathcal{R}^{\prime}(n, t)\right|=\left(1-2^{1-t}\right) \frac{q^{n}(\log n)^{t-1}}{(t-1) ! n}+O\left(\frac{q^{n}(\log n)^{t-2}}{n}\right) .
$$

\section{Proof of Theorem 1.1}

Let $F$ be an inert imaginary quadratic function field with $D_{F}=\gamma P_{1} \cdots P_{t}$. Let $s_{F}$ be the number of monic irreducible divisors $P_{i}$ of $D_{F}$ of odd degree. Since 
$\operatorname{deg} D_{F}$ is even, $s_{F}$ is an nonnegative even integer. Assume that $\operatorname{deg} P_{i}$ is odd for $1 \leq i \leq s_{F}$. Write $\vec{R}_{i}$ for the $i$-th row vector of $R_{F}$ for $1 \leq i \leq t$ and $\overrightarrow{0}$ for the zero row vector. By Schoof's Theorem, the Hilbert 2-class field tower of $F$ is infinite if $r_{2}\left(\mathcal{C l}_{F}\right) \geq 5$. Hence, it remains to prove the cases $\left(r_{2}\left(\mathcal{C} l_{F}\right), r_{4}\left(\mathcal{C} l_{F}\right)\right)=(4,4)$ if $s_{F}=0$ and $\left(r_{2}\left(\mathcal{C} l_{F}\right), r_{4}\left(\mathcal{C} l_{F}\right)\right)=(3,3),(4,3)$ or $(4,4)$ if $s_{F} \geq 2$.

3.1. Case $r_{2}\left(\mathcal{C} l_{F}\right)=r_{4}\left(\mathcal{C} l_{F}\right)=4$ with $s_{F}=0$ and $D_{F}=\gamma P_{1} P_{2} P_{3} P_{4}$. In this case, by $(2.1), R_{F}$ is a zero matrix, so $\left(\frac{P_{1}}{P_{i}}\right)=\left(\frac{P_{2}}{P_{i}}\right)=1$ for $i=3,4$. Hence $F$ has an infinite Hilbert 2-class field tower by Lemma 2.2 .

3.2. Case $r_{2}\left(\mathcal{C l} l_{F}\right)=r_{4}\left(\mathcal{C} l_{F}\right)=3$ with $s_{F} \geq 2$ and $D_{F}=\gamma P_{1} P_{2} P_{3} P_{4}$. In this case, we have $\operatorname{rank} R_{F}=1$ by $(2.1)$. Since $\vec{R}_{5} \neq \overrightarrow{0}$, we have $\vec{R}_{i} \in\left\{\overrightarrow{0}, \vec{R}_{5}\right\}$ for $1 \leq i \leq 4$. But, since $\sum_{j=1}^{4} e_{1 j}=1, \vec{R}_{1} \neq \overrightarrow{0}$ and $\vec{R}_{1} \neq \vec{R}_{5}$, which is a contradiction. Hence this case can not occur.

3.3. Case $r_{2}\left(\mathcal{C} l_{F}\right)=4, r_{4}\left(\mathcal{C} l_{F}\right)=3$ with $s_{F} \geq 2$ and $D_{F}=\gamma P_{1} P_{2} P_{3} P_{4} P_{5}$. In this case, we have $\operatorname{rank} R_{F}=2$ by $(2.1)$. Note that $\vec{R}_{6} \neq \overrightarrow{0}$. Since $\sum_{j=1}^{5} e_{1 j}=1$, we have $\vec{R}_{1} \notin\left\{\overrightarrow{0}, \vec{R}_{6}\right\}$. Then $\left\{\vec{R}_{1}, \vec{R}_{6}\right\}$ is a basis of the row space of $R_{F}$. Hence $\vec{R}_{i} \in$ $\left\{\overrightarrow{0}, \vec{R}_{1}, \vec{R}_{6}, \vec{R}_{1}+\vec{R}_{6}\right\}$ for all $1 \leq i \leq 5$. Assume that $s_{F}=2$. Then $\vec{R}_{6}=\left(\begin{array}{lllll}1 & 1 & 0 & 0 & 0\end{array}\right)$. If $\vec{R}_{i}=\overrightarrow{0}$ for some $3 \leq i \leq 5$, say $\vec{R}_{5}=\overrightarrow{0}$, then $\left(\frac{P_{5}}{P_{i}}\right)=1$ for $1 \leq i \leq 4$, so $F$ has infinite 2-class field tower by Lemma 2.1. We may assume $\vec{R}_{i} \neq \overrightarrow{0}$ for all $3 \leq i \leq 5$. Since $\sum_{j=1}^{5} e_{i j}=0$ for $3 \leq i \leq 5$, we have $\vec{R}_{3}=\vec{R}_{4}=\vec{R}_{5}=\vec{R}_{6}$. Then we have $\left(\frac{P_{3} P_{4}}{P_{i}}\right)=\left(\frac{P_{3} P_{5}}{P_{i}}\right)=1$ for $i=1,2$, so $F$ has infinite Hilbert 2-class field tower by Lemma 2.2.

Assume that $s_{F}=4$. Then $\vec{R}_{6}=\left(\begin{array}{lllll}1 & 1 & 1 & 1 & 0\end{array}\right)$. If $\vec{R}_{5}=\overrightarrow{0}$, then $\left(\frac{P_{5}}{P_{i}}\right)=1$ for $1 \leq i \leq 4$, so $F$ has infinite 2 -class field tower by Lemma 2.1. We may assume $\vec{R}_{5} \neq \overrightarrow{0}$. Since $\sum_{j=1}^{5} e_{5 j}=0$, we have $\vec{R}_{5}=\vec{R}_{6}$. Since $\sum_{j=1}^{5} e_{i j}=1$ for $1 \leq i \leq 4$, we have $\vec{R}_{i} \in\left\{\vec{R}_{1}, \vec{R}_{1}+\vec{R}_{6}\right\}$. If three of $\vec{R}_{1}, \vec{R}_{2}, \vec{R}_{3}, \vec{R}_{4}$ are equal, say $\vec{R}_{1}=\vec{R}_{2}=\vec{R}_{3}$, then $\left(\frac{P_{1} P_{2}}{P_{i}}\right)=\left(\frac{P_{1} P_{3}}{P_{i}}\right)=1$ for $i=4,5$, so $F$ has infinite Hilbert 2-class field tower by Lemma 2.2. We may assume that $\vec{R}_{1}=\vec{R}_{2}$ and $\vec{R}_{3}=\vec{R}_{4}=\vec{R}_{1}+\vec{R}_{6}$. Then, by the quadratic reciprocity law ([5, Theorem 3.3]), we have $e_{11}+e_{12}=1, e_{13}=e_{14}=e_{11}$ and $e_{15}=1$, so $\sum_{j=1}^{5} e_{1 j}=0$, which is a contradiction.

3.4. Case $r_{2}\left(\mathcal{C} l_{F}\right)=r_{4}\left(\mathcal{C} l_{F}\right)=4$ with $s_{F} \geq 2$ and $D_{F}=\gamma P_{1} P_{2} P_{3} P_{4} P_{5}$. In this case, we have $\operatorname{rank} R_{F}=1$ by (2.1). Since $\vec{R}_{6} \neq \overrightarrow{0}$, we have $\vec{R}_{i} \in\left\{\overrightarrow{0}, \vec{R}_{6}\right\}$ for $1 \leq i \leq 5$. But, since $\sum_{j=1}^{5} e_{1 j}=1, \vec{R}_{1} \neq \overrightarrow{0}$ and $\vec{R}_{1} \neq \vec{R}_{6}$, which is a contradiction. Hence this case can not occur. 


\section{Proof of Theorem 1.2}

For any positive even integer $n$ and positive integer $t$, let $\mathcal{R}(n, t)=\mathcal{P}(n, t) \backslash$ $\mathcal{P}_{2}(n, t)$ and $\mathcal{R}^{\prime}(n, t)=\mathcal{P}^{\prime}(n, t) \backslash \mathcal{P}_{2}^{\prime}(n, t)$.

Lemma 4.1. As $n \rightarrow \infty$, we have $\left|\mathcal{R}(n, t+1) \cup \mathcal{P}_{2}(n, t)\right| \sim\left|\mathcal{R}^{\prime}(n, t+1)\right|$.

Proof. By (2.4), (2.5) and (2.7), as $n \rightarrow \infty$, we have

$$
\begin{array}{ll}
\left|\mathcal{P}_{2}(n, t)\right|=O\left(\frac{q^{n}(\log n)^{t-1}}{n}\right), & \left|\mathcal{P}_{2}(n, t) \backslash \mathcal{P}_{2}^{\prime}(n, t)\right|=o\left(\frac{q^{n}(\log n)^{t-1}}{n}\right), \\
\left|\mathcal{R}^{\prime}(n, t+1)\right|=O\left(\frac{q^{n}(\log n)^{t}}{n}\right), & \left|\mathcal{R}(n, t+1) \backslash \mathcal{R}^{\prime}(n, t+1)\right|=o\left(\frac{q^{n}(\log n)^{t}}{n}\right) .
\end{array}
$$

Then $\left|\mathcal{P}_{2}(n, t) \backslash \mathcal{P}_{2}^{\prime}(n, t)\right|=o\left(\left|\mathcal{P}_{2}^{\prime}(n, t)\right|\right)$ and $\left|\mathcal{R}(n, t+1) \backslash \mathcal{R}^{\prime}(n, t+1)\right|=o\left(\mid \mathcal{R}^{\prime}(n, t+\right.$ $1) \mid)$. Also by $(2.7),\left|\mathcal{P}_{2}^{\prime}(n, t)\right|=o\left(\left|\mathcal{R}^{\prime}(n, t+1)\right|\right)$. Hence we get the result.

Recall that for an inert imaginary quadratic function field $F, r_{2}\left(\mathrm{Cl}_{F}\right)$ is equal to $\omega\left(D_{F}\right)$ or $\omega\left(D_{F}\right)-1$ according as $D_{F}$ is special or non-special. Let $n$ be a positive even integer and $r, s$ be integers with $0 \leq s \leq r$. Then we have

$$
Z_{r ; n}=\left\{k(\sqrt{\gamma N}): N \in \mathcal{R}(n, r+1) \cup \mathcal{P}_{2}(n, r)\right\} .
$$

Let $\bar{Z}_{r ; n}$ be the subset of $Z_{r ; n}$ consisting of $k(\sqrt{\gamma N})$ with $N \in \mathcal{R}^{\prime}(n, r+1)$ and $\bar{Z}_{r, s ; n}^{*}=\bar{Z}_{r ; n} \cap Z_{r, s ; n}^{*}$. By Lemma 4.1, we have

$$
\varrho_{r, s}^{*}=\liminf _{n \rightarrow \infty} \frac{\left|\bar{Z}_{r, s ; n}^{*}\right|}{\left|\bar{Z}_{r ; n}\right|} .
$$

By (2.7), we have

$$
\left|\bar{Z}_{r ; n}\right|=\left(1-2^{-r}\right) \frac{q^{n}(\log n)^{r}}{r ! n}+O\left(\frac{q^{n}(\log n)^{r-1}}{n}\right)
$$

as $n \rightarrow \infty$. For any $N \in \mathcal{R}^{\prime}(n, r+1)$, let $\mathcal{S}(N)$ be the set of inert imaginary quadratic function fields $k(\sqrt{\gamma M})$ with $M \in \mathcal{N}(N)$. Then $\mathcal{S}(N)$ is a subset of $\bar{Z}_{r ; n}$ and by (2.6), we have

$$
|\mathcal{S}(N)|=2^{-\frac{r(r+3)}{2}} \cdot \frac{q^{n}(\log n)^{r}}{r ! n}+O\left(\frac{q^{n}(\log n)^{r-1}}{n}\right)
$$

as $n \rightarrow \infty$. Thus, from (4.2) and (4.3), we get

$$
\lim _{n \rightarrow \infty} \frac{|\mathcal{S}(N)|}{\left|\bar{Z}_{r ; n}\right|}=2^{-\frac{r(r+1)}{2}}\left(1-2^{-r}\right)^{-1} .
$$


4.1. $\varrho_{3, s}^{*}$ Consider an inert imaginary quadratic function field $F=k(\sqrt{\gamma N})$ with $N=P_{1} P_{2} P_{3} P_{4} \in \mathcal{R}^{\prime}(n, 4)$ such that $2 \nmid \operatorname{deg} P_{i}, 2 \mid \operatorname{deg} P_{j}$ and $\left(\frac{P_{i}}{P_{j}}\right)=1$ for $i \in\{1,2\}$ and $j \in\{3,4\}$. Then $r_{2}\left(\mathcal{C l}_{F}\right)=3$ and $F$ has infinite Hilbert 2-class field tower by Lemma 2.2. Moreover, every fields in $\mathcal{S}(N)$ also has infinite Hilbert 2-class field tower.

- Case $\left(\frac{P_{3}}{P_{4}}\right)=-1$. In this case, the matrix $R_{F}$ is

$$
\left(\begin{array}{llll}
1 & 0 & 0 & 0 \\
1 & 0 & 0 & 0 \\
0 & 0 & 1 & 1 \\
0 & 0 & 1 & 1 \\
1 & 1 & 0 & 0
\end{array}\right) \text { if }\left(P_{1} / P_{2}\right)=1, \quad\left(\begin{array}{cccc}
0 & 1 & 0 & 0 \\
0 & 1 & 0 & 0 \\
0 & 0 & 1 & 1 \\
0 & 0 & 1 & 1 \\
1 & 1 & 0 & 0
\end{array}\right) \text { if }\left(P_{1} / P_{2}\right)=-1
$$

whose rank is 3 , so $r_{4}\left(\mathcal{C} l_{F}\right)=1$. Hence $F \in \bar{Z}_{3,1 ; n}^{*}$ and $\mathcal{S}(N) \subset \bar{Z}_{3,1 ; n}^{*}$. By (4.4), we have

$$
\varrho_{3,1}^{*} \geq \lim _{n \rightarrow \infty} \frac{|\mathcal{S}(N)|}{\left|\bar{Z}_{3 ; n}\right|}=2^{-3}\left(2^{3}-1\right)^{-1} .
$$

- Case $\left(\frac{P_{3}}{P_{4}}\right)=1$. In this case, the matrix $R_{F}$ is

$$
\left(\begin{array}{cccc}
1 & 0 & 0 & 0 \\
1 & 0 & 0 & 0 \\
0 & 0 & 0 & 0 \\
0 & 0 & 0 & 0 \\
1 & 1 & 0 & 0
\end{array}\right) \text { if }\left(P_{1} / P_{2}\right)=1, \quad\left(\begin{array}{cccc}
0 & 1 & 0 & 0 \\
0 & 1 & 0 & 0 \\
0 & 0 & 0 & 0 \\
0 & 0 & 0 & 0 \\
1 & 1 & 0 & 0
\end{array}\right) \text { if }\left(P_{1} / P_{2}\right)=-1
$$

whose rank is 2 , so $r_{4}\left(\mathcal{C} l_{F}\right)=2$. Hence $F \in \bar{Z}_{3,2 ; n}^{*}$ and $\mathcal{S}(N) \subset \bar{Z}_{3,2 ; n}^{*}$. By (4.4), we have

$$
\varrho_{3,2}^{*} \geq \lim _{n \rightarrow \infty} \frac{|\mathcal{S}(N)|}{\left|\bar{Z}_{3 ; n}\right|}=2^{-3}\left(2^{3}-1\right)^{-1}
$$

4.2. $\varrho_{4, s}^{*}$ Consider an inert imaginary quadratic function field $F=k(\sqrt{\gamma N})$ with $N=P_{1} P_{2} P_{3} P_{4} P_{5} \in \mathcal{R}^{\prime}(n, 5)$ such that $2 \nmid \operatorname{deg} P_{i}$ for $i \in\{1,2\}, 2 \mid \operatorname{deg} P_{j}$ for $j \in$ $\{3,4,5\}$ and $\left(\frac{P_{i}}{P_{j}}\right)=1$ for $i \in\{1,2\}, j \in\{3,4\}$. Then $r_{2}\left(\mathcal{C} l_{F}\right)=4$ and $F$ has infinite Hilbert 2-class field tower by Lemma 2.2. Moreover, every fields in $\mathcal{S}(N)$ also has infinite Hilbert 2-class field tower.

- Case $\left(\frac{P_{i}}{P_{5}}\right)=-1$ for $i \in\{1,3\},\left(\frac{P_{i}}{P_{5}}\right)=1$ for $i \in\{2,4\}$ and $\left(\frac{P_{3}}{P_{4}}\right)=-1$. In this case, the matrix $R_{F}$ is

$$
\left(\begin{array}{ccccc}
0 & 0 & 0 & 0 & 1 \\
1 & 0 & 0 & 0 & 0 \\
0 & 0 & 0 & 1 & 1 \\
0 & 0 & 1 & 1 & 0 \\
1 & 0 & 1 & 0 & 0 \\
1 & 1 & 0 & 0 & 0
\end{array}\right) \text { if }\left(P_{1} / P_{2}\right)=1, \quad\left(\begin{array}{ccccc}
1 & 1 & 0 & 0 & 1 \\
0 & 1 & 0 & 0 & 0 \\
0 & 0 & 0 & 1 & 1 \\
0 & 0 & 1 & 1 & 0 \\
1 & 0 & 1 & 0 & 0 \\
1 & 1 & 0 & 0 & 0
\end{array}\right) \text { if }\left(P_{1} / P_{2}\right)=-1
$$

whose rank is 5 , so $r_{4}\left(\mathcal{C} l_{F}\right)=0$. Hence $F \in \bar{Z}_{4,0 ; n}^{*}$ and $\mathcal{S}(N) \subset \bar{Z}_{4,0 ; n}^{*}$. By (4.4), we have

$$
\varrho_{4,0}^{*} \geq \lim _{n \rightarrow \infty} \frac{|\mathcal{S}(N)|}{\left|\bar{Z}_{4 ; n}\right|}=2^{-6}\left(2^{4}-1\right)^{-1} .
$$


- Case $\left(\frac{P_{1}}{P_{5}}\right)=-1,\left(\frac{P_{i}}{P_{5}}\right)=1$ for $2 \leq i \leq 4$ and $\left(\frac{P_{3}}{P_{4}}\right)=-1$. In this case, the matrix $R_{F}$ is

$$
\left(\begin{array}{lllll}
0 & 0 & 0 & 0 & 1 \\
1 & 0 & 0 & 0 & 0 \\
0 & 0 & 1 & 1 & 0 \\
0 & 0 & 1 & 1 & 0 \\
1 & 0 & 0 & 0 & 1 \\
1 & 1 & 0 & 0 & 0
\end{array}\right) \text { if }\left(P_{1} / P_{2}\right)=1, \quad\left(\begin{array}{ccccc}
1 & 1 & 0 & 0 & 1 \\
0 & 1 & 0 & 0 & 0 \\
0 & 0 & 1 & 1 & 0 \\
0 & 0 & 1 & 1 & 0 \\
1 & 0 & 0 & 0 & 1 \\
1 & 1 & 0 & 0 & 0
\end{array}\right) \text { if }\left(P_{1} / P_{2}\right)=-1
$$

whose rank is 4 , so $r_{4}\left(\mathcal{C} l_{F}\right)=1$. Hence $F \in \bar{Z}_{4,1 ; n}^{*}$ and $\mathcal{S}(N) \subset \bar{Z}_{4,1 ; n}^{*}$. By (4.4), we have

$$
\varrho_{4,1}^{*} \geq \lim _{n \rightarrow \infty} \frac{|\mathcal{S}(N)|}{\left|\bar{Z}_{4 ; n}\right|}=2^{-6}\left(2^{4}-1\right)^{-1}
$$

- Case $\left(\frac{P_{i}}{P_{5}}\right)=1$ for $1 \leq i \leq 4$ and $\left(\frac{P_{3}}{P_{4}}\right)=-1$. In this case, the matrix $R_{F}$ is

$$
\left(\begin{array}{lllll}
1 & 0 & 0 & 0 & 0 \\
1 & 0 & 0 & 0 & 0 \\
0 & 0 & 1 & 1 & 0 \\
0 & 0 & 1 & 1 & 0 \\
0 & 0 & 0 & 0 & 0 \\
1 & 1 & 0 & 0 & 0
\end{array}\right) \text { if }\left(P_{1} / P_{2}\right)=1, \quad\left(\begin{array}{ccccc}
0 & 1 & 0 & 0 & 0 \\
0 & 1 & 0 & 0 & 0 \\
0 & 0 & 1 & 1 & 0 \\
0 & 0 & 1 & 1 & 0 \\
0 & 0 & 0 & 0 & 0 \\
1 & 1 & 0 & 0 & 0
\end{array}\right) \text { if }\left(P_{1} / P_{2}\right)=-1
$$

whose rank is 3 , so $r_{4}\left(\mathcal{C l}_{F}\right)=2$. Hence $F \in \bar{Z}_{4,2 ; n}^{*}$ and $\mathcal{S}(N) \subset \bar{Z}_{4,2 ; n}^{*}$. By (4.4), we have

$$
\varrho_{4,2}^{*} \geq \lim _{n \rightarrow \infty} \frac{|\mathcal{S}(N)|}{\left|\bar{Z}_{4 ; n}\right|}=2^{-6}\left(2^{4}-1\right)^{-1} .
$$

- Case $\left(\frac{P_{i}}{P_{5}}\right)=1$ for $1 \leq i \leq 4$ and $\left(\frac{P_{3}}{P_{4}}\right)=1$. In this case, the matrix $R_{F}$ is

$$
\left(\begin{array}{lllll}
1 & 0 & 0 & 0 & 0 \\
1 & 0 & 0 & 0 & 0 \\
0 & 0 & 0 & 0 & 0 \\
0 & 0 & 0 & 0 & 0 \\
0 & 0 & 0 & 0 & 0 \\
1 & 1 & 0 & 0 & 0
\end{array}\right) \text { if }\left(P_{1} / P_{2}\right)=1, \quad\left(\begin{array}{ccccc}
0 & 1 & 0 & 0 & 0 \\
0 & 1 & 0 & 0 & 0 \\
0 & 0 & 0 & 0 & 0 \\
0 & 0 & 0 & 0 & 0 \\
0 & 0 & 0 & 0 & 0 \\
1 & 1 & 0 & 0 & 0
\end{array}\right) \text { if }\left(P_{1} / P_{2}\right)=-1
$$

whose rank is 2 , so $r_{4}\left(\mathcal{C l}_{F}\right)=3$. Hence $F \in \bar{Z}_{4,3 ; n}^{*}$ and $\mathcal{S}(N) \subset \bar{Z}_{4,3 ; n}^{*}$. By (4.4), we have

$$
\varrho_{4,3}^{*} \geq \lim _{n \rightarrow \infty} \frac{|\mathcal{S}(N)|}{\left|\bar{Z}_{4 ; n}\right|}=2^{-6}\left(2^{4}-1\right)^{-1}
$$

\section{REFERENCES}

1. S. Bae, S. Hu \& H. Jung: The generalized Rédei matrix for function fields. Finite Fields and Their Applications 18 (2012), no 4, 760-780.

2. H. Jung: Hilbert 2-class field towers of imaginary quadratic function fields. submitted.

3 . : Hilbert 2-class field towers of real quadratic function fields. submitted.

4. M. Rosen: The Hilbert class field in function fields. Exposition. Math. 5 (1987), no. 4, 365-378.

5. __ N N Number theory in function fields. Graduate Texts in Mathematics, 210. SpringerVerlag, New York, 2002. 
6. R. Schoof: Algebraic curves over $\mathbb{F}_{2}$ with many rational points. J. Number Theory 41 (1992), no. 1, 6-14.

Department of Mathematics Education, Chungbuk National University, Cheonguu 361763, Korea

Email address: hyjung@chungbuk.ac.kr 